\title{
On the Mechanism of Polyuria in Potassium Depletion
}

\author{
THE ROLE OF POLYDIPSIA
}

\author{
Tomas Berl, Stuart L. Linas, Gary A. Aisenbrey, and Robert J. Anderson \\ From the Division of Renal Diseases, Department of Medicine, University of Colorado Medical \\ Center, Denver, Colorado 80262
}

A в S T R A C T The association of potassium (K) depletion with polyuria and a concentrating defect is established, but the extent to which these defects could be secondary to an effect of low $\mathrm{K}$ on water intake has not been systematically investigated. To determine whether hypokalemia has a primary effect to increase thirst and whether any resultant polyuria and polydipsia contribute to the concentrating defect, we studied three groups of rats kept in metabolic cages for 15 days. The groups were set up as follows: group 1, normal diets and ad lib. fluids $(n=12)$; group 2, K-deficient diet on ad lib. fluids $(n=12)$; and group 3, K-deficient diet and fluid intake matched to group $1(n=14)$. Daily urine flow and urinary osmolality of groups 1 and 3 were not significantly different throughout the study. In contrast, as of day 6 , group 2 rats consistently had a higher fluid intake $(P<0.0025)$, higher urine flow $(P<0.001)$, and lower urinary osmolality $(P<0.001)$ than the other two groups. These alterations in fluid intake and urine flow preceded a defect in maximal concentrating ability. On day 7, maximal urinary osmolality was $2,599 \pm 138 \mathrm{msmol} / \mathrm{kg}$ in rats on K-deficient intake and $2,567 \pm 142 \mathrm{msmol} / \mathrm{kg}$ in controls. To determine whether this primary polydipsia is itself responsible for the development of the concentrating defect, the three groups of rats were dehydrated on day 15 . Despite different levels of fluid intake, maximal urinary osmolality was impaired equally in groups 2 and 3 $(1,703$ and $1,511 \mathrm{msmol} / \mathrm{kg}$, respectively), as compared to rats in group $1(2,414 \mathrm{msmol} / \mathrm{kg}), P<0.001$. We therefore conclude that $\mathrm{K}$ depletion stimulates thirst, and the resultant increase in water intake is largely responsible for the observed polyuria. After 15 days of a K-deficient diet, the impaired maximal urinary concentration in hypokalemia, however, was

Received for publication 9 November 1976 and in revised form 26 April 1977. not related to increased water intake, since fluid restriction did not abolish the renal concentrating defect.

\section{INTRODUCTION}

The association of hypokalemia with polyuria and a renal concentrating defect has been well established in both man (1) and experimental animals $(2,3)$. Studies in experimental animals have suggested that these derangements may be partially due to a decrease in medullary tonicity (2). The mechanism responsible for the decrease in medullary tonicity remains controversial and derangements in both the function of the proximal tubule (4) and the ascending loop of Henle (5) have been proposed. A similar washout of medullary tonicity also occurs with excessive water intake and secondary polyuria (6), and may be responsible for the concentrating defect noted in psychogenic polydipsia (7). It, therefore, follows that the polyuria and the renal concentrating defect of hypokalemia could be a consequence of increased thirst and water intake. In this regard, early observers noted that both dogs (8) and rats (9) increased their water intake soon after being placed on a potassium (K)-deficient diet. The pathogenetic relationships between such increased water intake and the polyuria and renal concentrating defect in the hypokalemic state have not been fully defined. The present study thus was undertaken to establish whether hypokalemia has a primary effect on thirst and, if so, to determine whether the ensuing polydipsia contributes to the polyuria and/or to the development of the renal concentrating defect.

\section{METHODS}

All studies were performed on 78 Sprague-Dawley rats weighing between 250 and $325 \mathrm{~g}$ and maintained in metabolic cages that allow food, urine, and feces separation (Holtge 
Co., Cincinnati, Ohio). Animals were allowed $48 \mathrm{~h}$ to adapt to the cages before study collections were started. Kdeficient diet ( $5 \mathrm{meq} / \mathrm{kg}$ food) was commercially obtained from ICN Nutritional Biochemicals Div., International Chemical \& Nuclear Corp., Cleveland, Ohio, and was supplemented with magnesium. The diet consumed by control animals was prepared by adding a combination of $K$ salts to the above diet to render the $K$ concentration equal to that of regular chow (232) meq/ $\mathrm{kg}$ food). The following studies were undertaken.

Comparison of water intake in animals on K-supplemented and $\mathrm{K}$-deficient diets and its temporal relation to impaired urinary concentration. The daily water intake, urine output, urinary osmolality, and weight of rats of K-supplemented (group 1) and K-deficient (group 2) diets were compared for 15 consecutive days ( $n=12$ in each group). The same comparisons were obtained in five rats in each group who were pair fed. Such feeding was accomplished by allowing rats on the K-supplemented intake no more food than consumed by its pair fed experimental animal in the previous $24 \mathrm{~h}$. The rats on the K-supplemented diet uniformly consumed all the food given to them. In two separate groups of identically treated K-deficient and K-supplemented animals ( $n=15$ in each group, 5 of which were pair fed), the ability to maximally concentrate the urine was determined after a 24-h dehydration on day 7 , the time by which the K-deficient animals had an intake clearly greater than control rats. To ensure maximal levels of circulating vasopressin during the concentrating test, five rats in each group were also given a subcutaneous injection of 500 $\mathrm{mU}$ of antidiuretic hormone in oil on the day of dehydration and the injection was repeated the next morning when the final urine was being obtained.

Effect of water restriction on urine flow, urinary osmolality, and weights in animals on low $K$ intake. The extent to which an increase in water intake could contribute to the polyuria of $\mathrm{K}$ depletion was determined by simultaneously studying a group of 14 rats (group 3) on Kdeficient diet whose water intake was matched daily to the previous day's intake of rats on K-supplemented diet (group 1). The daily fluid volume was offered in three separate aliquots. These animals' urine output urinary osmolality, and weight were determined daily.

Role of polydipsia and polyuria in the development of a concentrating defect. The contribution of polyuria and polydipsia to the concentrating defect in hypokalemia was determined by comparing the maximal urinary osmolality of hypokalemic rats allowed free water access (group 2, $n=12$ ) to that of equally hypokalemic rats who were water restricted (group $3, n=14$ ) and, therefore, not allowed to develop polyuria and polydipsia. After 15 days in metabolic cages, these rats, as well as normokalemic controls (group $1, n=12$ ), underwent a 24-h concentrating test as described above. Animals were then sacrificed. Serum urea nitrogen, creatinine (Technicon AutoAnalyzer, Technicon Corp., Tarrytown, N. Y.), sodium, and K (Flame photometer) were then determined. Urinary osmolality was measured by freezing point depression.

Statistical analysis was performed by unpaired $t$ test when two groups of animals were compared. The three groups were compared by one-way analysis of variance. Individual comparisons were made separately and cumulatively each day and multivariately across all 15 days to summarize the results. A $P$ value $<0.05$ was considered not significant.

\section{RESULTS}

Water intake in rats on $K$-supplemented and $K$ deficient diets and its temporal relation to impaired urinary concentration (Fig. 1). The water intake of control rats on K-supplemented intake (group 1) was essentially unaltered in the 15 days of the study. As noted in Fig. 1, the mean water intake of rats on the K-deficient diet (group 2) was not significantly different from controls in the first 5 days. The difference became significant on day 6 and remained so thereafter. The cumulative difference in water intake over the 15 days was significant to the $P<0.0025$ level.

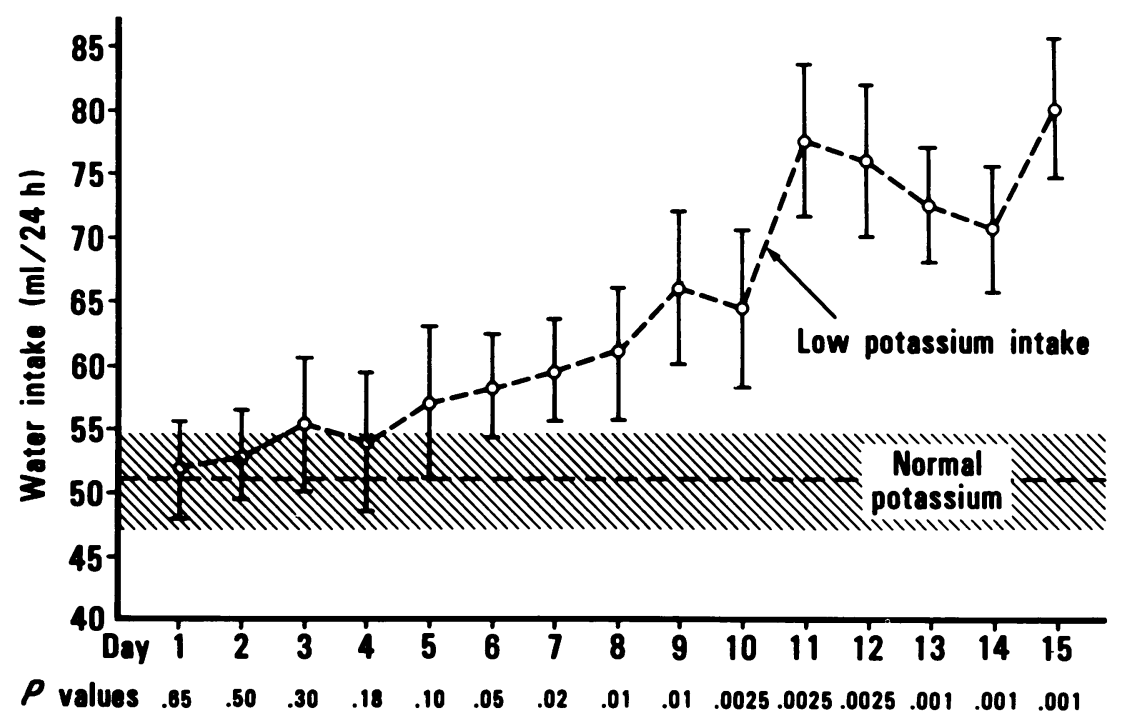

FIGURE 1 Mean $\pm S E$ daily water intake of rats on a low potassium diet $(n=12)$ compared to the mean $\pm \mathrm{SE}$ water intake of rats on normal potassium diet $(n=12)$, depicted by the shaded area. 
Rats on the K-free diet consumed less food, as reflected in a significantly lower daily solute excretion (see below). Since such a decrease in food intake could in itself alter drinking behavior, the observations were repeated by pair feeding five rats on a $\mathrm{K}$ supplemented diet to an equal number of animals on K-deficient food. These pair fed animals had almost identical solute excretions, $26.7 \pm 1.6$ and $27.4 \pm 1.9$ mosmol/24 h, respectively. The pair feeding did not, however, alter the pattern of water intake noted above. In fact, the difference in water intake was already significant on day $4(P<0.01)$ and by day 6 the difference was significant to the $P<0.001$ level.

A 24-h concentrating test was performed on 15 rats on K-supplemented intake and 15 rats on K-deficient diet for 7 days. Five of the rats in each group underwent this dehydration in the presence of large doses of vasopressin. The maximal urinary concentration achieved was not increased by the hormone in either group. No significant impairment in maximal urinary osmolality was noted at this time as mean urinary osmolalities were $2567 \pm 142$ and $2,599 \pm 138 \mathrm{msmol} / \mathrm{kg}$ $\mathrm{H}_{2} \mathrm{O}$ in rats on K-supplemented and K-deficient intake, respectively. The weight loss sustained during the dehydration and the serum sodium after the test were indistinguishable in the two groups. Plasma K at this time was significantly decreased in animals on K-deficient diets $(2.54 \pm 0.01$ vs. $3.43 \pm 0.4 \mathrm{meq} / \mathrm{liter}$ in controls, $P<0.001$ ), but this level was still higher than the serum $\mathrm{K}$ measured after 15 days on the diet, $2.05 \pm 0.05 \mathrm{meq} / \mathrm{liter}(P<0.001)$. These results demonstrate that an increase in water intake clearly precedes the development of a concentrating defect in the rat developing $\mathbf{K}$ depletion.

Effect of water restriction on urine flow, urinary osmolality, and weights in animals on low $\mathrm{K}$ intake (Figs. 2 and 3, Table I). To determine the role of polydipsia on the polyuria of $\mathrm{K}$ depletion, daily urinary osmolality and urine flow were determined in animals on low-K diet whose intake was restricted to that of control rats on K-supplemented diets. As depicted in Fig. 2 (top), such animals (denoted as group 3) had urine outputs that were not significantly different from animals on normal $\mathrm{K}$ intake (group 1 ). As of day 6, both control rats (group 1) and waterrestricted hypokalemic rats (group 3) had urine outputs consistently and significantly lower than that of rats on K-deficient intake allowed ad lib. fluids (group $2), P<0.001$. The increase in urine flow in this latter group is clearly not related to increased solute excretion. In fact, the mean solute excretion of these rats was $28.0 \pm 0.99 \mathrm{mosmol} / 24 \mathrm{~h}$, which is significantly lower than that of control rats, $31.8 \pm 0.8 \mathrm{mosmol} / 24 \mathrm{~h}$ $(P<0.01)$. This decrease in solute excretion was also observed in the water-restricted animals on low-K diet (group 3) whose mean solute excretion was 26.8 $\pm 0.56 \mathrm{mosmol} / 24 \mathrm{~h}$, not significantly different from animals on the same diet allowed ad lib. water (group 2) but lower than that of controls $(P<0.005)$. The changes in urine flow were reflected in daily urinary osmolality (Fig. 2, bottom). Although urinary osmolality was consistently lower in group 2 animals, the urinary concentration of animals with comparable water intake (groups 1 and 3) did not significantly differ regardless of the $\mathrm{K}$ content of their diet.

The ability of rats on a low-K intake, whose water intake was restricted (group 3), to decrease their urine flow and increase their urinary osmolality to control levels could have been due to intrarenal factors mediated by negative water on extracellular fluid balance. However, as shown in Fig. 3 and Table I, these rats had no significant alteration in body weight throughout the study and in this respect were at no time different from animals on the same diet allowed ad lib. fluid intake (group 2). In contrast to this previously noted failure of animals on K-restricted intake to grow (10), animals on normal intake grew throughout the study. This gain in weight was seen in rats on normal intake even when they were pair fed, although at a slower rate. Furthermore, the serum sodium, blood urea nitrogen, and creatinine of these water-restricted rats was not different from that of either control or K-restricted animals allowed free water access (Table I).

Effect of polydipsia and polyuria on the development of the concentrating defect of hypokalemia (Table I). To determine whether the polydipsia and polyuria associated with hypokalemia is in any way responsible for the concentrating defect that ensues in this disorder, the ability to maximally concentrate the urine in animals not allowed to become polydipsic and polyuric (group 3) was compared to that of equally hypokalemic rats allowed free water access (group 2). As noted in Table I, these two groups of rats became comparably hypokalemic with serum $\mathrm{K}$ of 2.05 and $2.16 \mathrm{meq} / \mathrm{liter}$, and they both developed an equally severe defect in urinary concentration, 1,703 $\pm 95 \mathrm{mosmol} / \mathrm{kg}$ for group 2 and $1,511 \pm 129 \mathrm{mosmol} / \mathrm{kg}$ for group 3, both significantly lower than controls in group $1,2,414 \pm 128 \mathrm{mosmol} / \mathrm{kg}, P<0.001$.

\section{DISCUSSION}

Although there is incontrovertible evidence that $\mathrm{K}$ depletion is associated with profound alterations in water homeostasis characterized by polyuria, polydipsia, and impaired maximal urinary concentration, neither the exact pathogenesis nor the temporal relation in which these defects supervene have been fully defined. The possibility that the primary event in this derangement is an effect of hypokalemia on thirst and water intake has not been systematically 


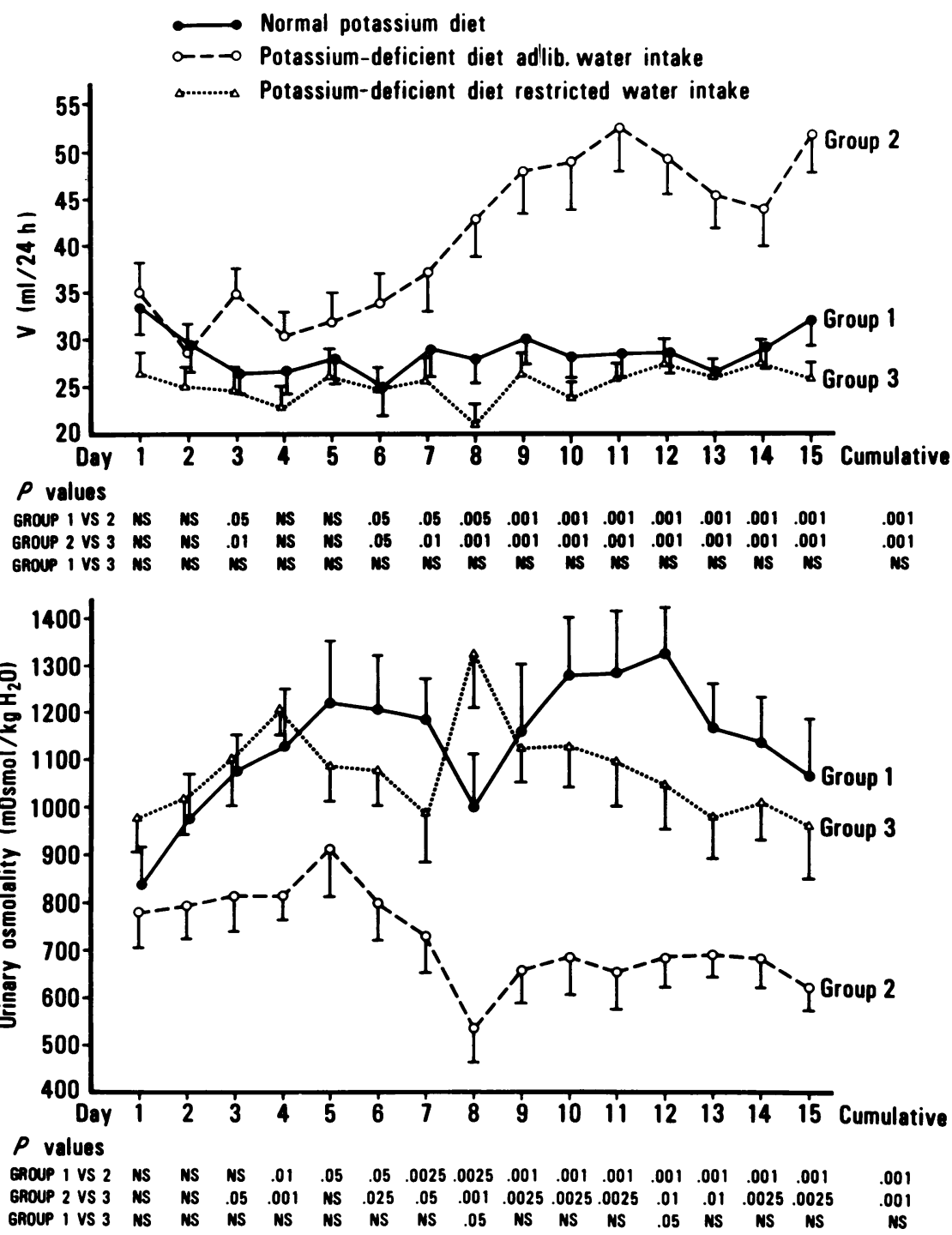

FIGURE 2 Mean urine flow (top) and mean daily urinary osmolality (bottom) of rats on normal potassium intake (group $1, n=12$ ), potassium-deficient diet on ad lib. fluid intake (group 2, $n=12$ ), and potassium-deficient intake with water intake matched to that of rats on normal potassium diet (group $3, n=14$ ).

evaluated. Such an effect could explain not only the observed polyuria but could itself account for the measured decrease in medullary tonicity that is associated with hypokalemia (2). In this regard, Smith and Lasater (8) in dogs and Brokaw (9) in rats observed that water intake increased promptly after animals were placed on $\mathrm{K}$-deficient intake. However, neither study reported on the osmolality of the excreted urine. Subsequently, Hollander et al. (10) noted that rats developed polydipsia almost immediately after being placed on a $\mathrm{K}$ depleted diet, their water intake being greater than that of their pair fed controls. However, since maximal urinary concentration was not tested at that early time, the authors acknowledge that the relationship of the polydipsia to a defect in the concentrating process cannot be ascertained from their data. Likewise, Kleeman (11) suspected, on the basis of his clinical observations, that $\mathrm{K}$ depletion may affect water intake in man as well.

The present study was designed to determine whether $\mathrm{K}$ depletion has an effect on thirst and, if so, whether such an effect is responsible for the deranged renal conservation of water. Our studies provide rather conclusive answers to both of these questions. It becomes evident from a comparison of drinking behavior that the water intake of animals placed on K-deficient diet is increased rather promptly, although the difference does not attain consistent statisti- 


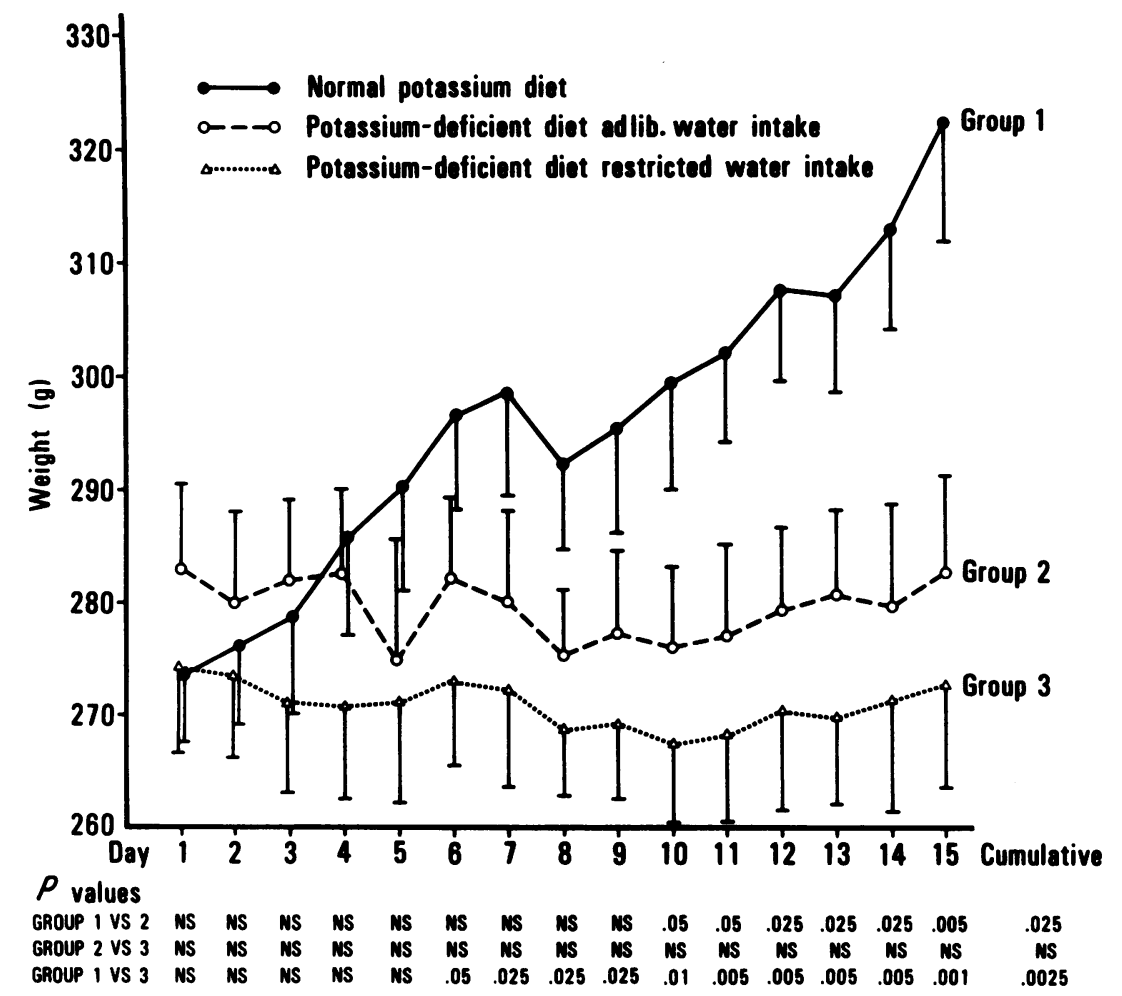

FIGURE 3 Daily weight of rats on normal potassium intake (group 1), potassium-deficient diet on ad lib. water intake (group 2), and potassium-deficient diet with water intake matched to that of rats on normal potassium diet (group 3). Whereas group 1 rats grew throughout the study, neither group 2 or 3 had changes in weight significantly different from 0 or from each other.

cal significance until day 6 (Fig. 1). This increase in water intake precedes the onset of a significant renal concentrating defect, since maximal urinary osmolality of rats fed K-deficient and K-supplemented diets was no different on day 7 , a time at which water intake was significantly higher in the animals receiving the low $\mathrm{K}$ intake. These observations could not be explained on the basis of differences in total caloric or

TABLE I

Effect of 15 Days of Potassium-Deficient Diet with and without Water Restriction on Body Weight, Serum Sodium, Potassium, Urea, Creatinine, and Maximal Urinary Osmolality

\begin{tabular}{|c|c|c|c|c|c|c|}
\hline Study group & $\begin{array}{c}\text { Change in } \\
\text { weight }\end{array}$ & $\mathrm{Na}$ & $\mathbf{K}$ & $\begin{array}{c}\text { Blood urea } \\
\text { nitrogen }\end{array}$ & Creatinine & $\begin{array}{l}\text { Maximal urinary } \\
\text { osmolality }\end{array}$ \\
\hline & $\mathrm{g}$ & \multicolumn{2}{|c|}{ meqliter } & \multicolumn{2}{|c|}{$m g / 100 \mathrm{ml}$} & mosmollkg \\
\hline $\begin{array}{l}\text { Group I }(n=12) \\
\text { K-supplemented diet } \\
\text { ad lib. water intake }\end{array}$ & $+47.1 \pm 6.2$ & $146.8 \pm 0.62$ & $3.48 \pm 0.10$ & $22.7 \pm 0.93$ & $0.59 \pm 0.04$ & $2,414 \pm 128$ \\
\hline $\begin{array}{l}\text { Group II }(n=12) \\
\text { K-deficient diet } \\
\text { ad lib. water intake }\end{array}$ & $-1.0 \pm 7.9$ & $147.9 \pm 0.52$ & $2.05 \pm 0.05$ & $24.1 \pm 1.4$ & $0.60 \pm 0.06$ & $1,511 \pm 129$ \\
\hline $\begin{array}{l}\text { Group III }(n=14) \\
\text { K-deficient diet } \\
\text { water intake matched to group I } \\
P \text { value }\end{array}$ & $-1.8 \pm 8.2$ & $147.8 \pm 0.61$ & $2.16 \pm 0.09$ & $23.5 \pm 1.1$ & $0.56 \pm 0.02$ & $1,703 \pm 95$ \\
\hline I-II & 0.001 & NS & 0.001 & NS & NS & 0.001 \\
\hline II-III & NS & NS & NS & NS & NS & NS \\
\hline I-III & 0.001 & NS & 0.001 & NS & NS & 0.001 \\
\hline
\end{tabular}


protein intake. In fact, in the pair fed experiments, the difference in water intake was observed even earlier whereas concentrating ability was unimpaired on day 7. In the above mentioned studies of Hollander et al. (10), a group of K-depleted rats, whose water intake was not monitored, had a mild concentrating defect $\left(2,132 \mathrm{mosmol} / \mathrm{kg} \mathrm{H}_{2} \mathrm{O}\right)$, when dehydrated after $1 \mathrm{wk}$ on the diet. However, these rats were not pair fed and their diet also contained $4 \%$ of urea, which, as the authors note, caused some of them to have diarrhea. The development of $\mathrm{K}$ depletion was thus accelerated in these rats as was also reflected by the fact that their muscle $\mathrm{K}$ content was as low after $1 \mathrm{wk}$ on the diet as that of rats on a similar K-deficient diet without supplemental urea after $3 \mathrm{wk}(10)$. In all likelihood, therefore, the small discrepancy in maximal urinary concentration is related to the greater degree of $\mathrm{K}$ depletion at the time of dehydration of rats in that study when compared to those of the present one. Our results, therefore, suggest that after 7 days on a Kdeficient diet, the excretion of a larger volume of urine of lower osmolality is a consequence of increased water intake rather than a renal concentrating defect. Likewise, the ability of hypokalemic animals to maintain urine output and daily urinary osmolality at a level comparable to control animals if their water intake is restricted, suggests that the polyuria of the hypokalemic animals allowed free water intake was a consequence of excessive intake throughout the study period. The ability to maintain this urine output and urinary osmolality in the water restricted hypokalemic rats was not achieved at the expense of water depletion, extraceliular fluid volume contraction, or decreased glomerular filtration rate as reflected by no difference in serum sodium concentration, serial weights, and blood urea nitrogen and creatinine measurements when compared to equally hypokalemic rats allowed free water access (Table I). Inasmuchas hypokalemic animals ate less and weighed less than controls, the comparable levels of blood urea nitrogen and creatinine may reflect a slight but equal decrease in glomerular filtration rate in the two groups of hypokalemic rats. This primary effect of $\mathrm{K}$ depletion to stimulate water intake probably explains the observation that the polyuria of hypokalemic subjects is often in excess of the urine volume obligated by their concentrating defect. The mechanism whereby the development of $\mathrm{K}$ depletion causes polydipsia is in need of further study, but since it precedes a defect in water conservation, the implication is that the stimulus to thirst is not hyperosmolality, but rather is of a nonosmolar nature.

Since polydipsia and polyuria can culminate in a vasopressin resistant impairment of urinary concentration (6), the possibility existed that the stimulation of thirst and water intake might be responsible for the renal concentrating defect. However, the development of a similar concentrating defect by day 15 in hypokalemic animals on restricted or ad lib. water intake demonstrates that the concentrating defect is independent of the high water intake in these rats, as was suggested by Blythe et al. (12).

In conclusion, the results of the present study indicate that $\mathrm{K}$ depletion affects water homeostasis by both central and renal mechanisms. The former is characterized by the stimulation of thirst and leads to a primary polydipsia which is in large measure responsible for the polyuria observed in this disorder. Further $\mathrm{K}$ depletion then culminates in a derangement of maximal urinary concentration which is independent of the polyuria and polydipsia and is therefore most likely due to a direct renal effect of hypokalemia.

\section{ACKNOWLEDGMENTS}

The authors are most grateful to Dr. Robert W. Schrier for his suggestions in the course of the study and his advice in the preparation of this manuscript. We also wish to thank Dr. Gary Zerbe in the Department of Biostatistics for assistance in the analysis of the data and to Ms. Linda M. Benson for expert secretarial assistance.

Work performed was under sponsorship of a National Institutes of Health grant, HL 15629.

\section{REFERENCES}

1. Rubini, M. E. 1961. Water excretion in potassium deficient man. J. Clin. Invest. 40: 2215-2224.

2. Mannitius, A., H. Levitin, D. Beck, and F. H. Epstein. 1960. On the mechanism of renal concentrating ability in potassium deficiencies. J. Clin. Invest. 39: 684-692.

3. Bennett, C. M. 1970. Urine concentration and dilution in hypokalemia and hypercalcemia dogs. J. Clin. Invest. 49: $1447-1457$.

4. Bank, N., and H. S. Ajnedjian. 1964. A micropuncture study of the renal concentrating defect of potassium depletion. Am. J. Physiol. 206: 1347-1354.

5. Eknoyan, G., M. Martinez-Maldonado, W. Suki, and Y. Richie. 1970. Renal diluting capacity in the hypokalemic rat. Am. J. Physiol. 219: 933-937.

6. Levitin, H., A. Goodman, G. Pigeon, and F. H. Epstein. 1962. Composition of the renal medulla during water diuresis. J. Clin. Invest. 41: 1145-1151.

7. Barlow, E. D., and H. E. de Wardener. 1959. Compulsive water drinking. Q. J. Med. 28: 235-258.

8. Smith, S. G., and T. Lasater. 1950. A diabetes insipiduslike condition produced in dogs on potassium deficient diet. Proc. Soc. Exp. Biol. Med. 74: 427. (Abstr.)

9. Brokaw, A. 1953. Renal hypertrophy and polydipsia in potassium deficient rats. Am. J. Physiol. 172: 333-346.

10. Hollander, W., R. W. Winters, F. Williams, J. Bradley, J. Oliver, and L. G. Welt. 1957. Defect in renal reabsorption of water associated with potassium depletion in rats. Am. J. Physiol. 189: 557-563.

11. Kleeman, C. R. 1972. Water metabolism. In Clinical Disorders of Fluid and Electrolyte Metabolism. M. H. Maxwell and C. R. Kleeman, editors. McGraw-Hill, Inc., New York. 2nd edition. 215-295.

12. Blythe, W. B., M. Newton, F. Lazcano, and L. G. Welt. 1960. Effect of water restriction on urinary concentration ability of K-depleted rats. Am. J. Physiol. 199: 912-914.

Potassium Depletion and Polydipsia 